\title{
The Polymer in a Cone-A Model for the Surface Free Energy of Polymer Crystal with Emergent Cilia
}

\author{
C. M. Guttman*, E. A. DiMarzio*, and J. D. Hoffman* \\ National Bureau of Standards, Washington, D.C. 20234
}

\begin{abstract}
March 12, 1980
A model is proposed to estimate the surface free energy of a small polymer crystal with numerous emergent cilia. For such a model the partition function of a polymer constrained to remain in a cone is computed. The partition function of the polymer in a cone is found to behave similarly to the polymer in a wedge discussed by Lauritzen and DiMarzio [1]. ${ }^{1}$ The estimated end surface free energy per unit area for the small extended chain crystal is found to increase with increasing area, implying the presence of cumulative surface stress in such crystals. The forces between the cilia are reduced if folds are inserted in the surface.
\end{abstract}

Key words: Constrained polymer; polymer; polymer in a cone; polymer interference; small polymer crystal; surface free energy.

\section{Introduction}

The effect of surfaces on polymer configurations has long been studied. For example, DiMarzio and Rubin [2] and Gaylord [3] have studied the modification of the configurational partition function of polymers resulting from a polymer being attached to an adsorbing surface or enclosed by reflecting walls. Helfand [4] has studied the interface between two different polymers in a block copolymer system while Dolan and Edwards [5] have studied the effect of a surface on random coils as well as on excluded volume coils. All the above mentioned works show significant effects on the polymer chains as a result of the presence of a surface.

Thus, when a polymer chain emanates from an ordered phase into a disordered phase-as in the case of a polymer chain partaking in both the crystalline and amorphous components of a semi-crystalline polymer-significant effects on the polymer configurations in the amorphous component are to be expected.

Flory [6] proposed a model for a polymer system of lamellar morphology in which each amorphous polymer chain is never allowed to step back towards the crystal region it left. This model permits only one-half to one-third (depending on geometry) of the chains from the crystal to transverse the amorphous regime but requires an entropy defect of approximately $N_{c} 1 n c / 2$ per chain, where $c$ is the coordination number of the monomer unit and $N_{c}$ is the number of

\footnotetext{
* National Measurement Laboratory

${ }^{1}$ Figures in brackets indicate literature references at the end of this paper.
}

segments in the cilia, for each chain emanating from the surface.

DiMarzio [7] developed a model in which the chains emanating from the surface were completely random coils and thus had no free energy defect. However this model allowed only $1 / \sqrt{ } N_{c}$ of the crystalline chains to emanate from the surface. More recently, Guttman and DiMarzio [8] have proposed models intermediate between the extreme of the DiMarzio and Flory models.

The aforementioned models assumed infinite extent of the plane from which the cilia emanate. Recently, Hoffman [9] and Keller [10] modeled the finite sized polymer crystal produced by Pennings stir crystallization [11]. The model of the so called Penning "shish" is shown in figure la. Hoffman has suggested that the chains emanating from the crystal end interfere with one another causing an increasing surface free energy per unit area as the crystal grows. This change in surface free energy is found to be large enough to cause the crystal to stop growing [9].

In this paper, we shall estimate the surface free energy of a finite size crystal with emanating cilia interfering with one another as a function of crystal size. Each crystal is assumed to be a right circular cylinder of known radius and length. As the diameter of the crystal grows, the chain portions emanating from the end of the cylinders interfere with one another more and more (see fig. $1 \mathrm{~b}$ ). We imagine the space allowed to all the fraying ends of the crystal to consist of a cone of angle $\Omega$. Each of the $n_{c}$ chain ends must stay within the angle $\Omega$ and compete with all the other chains for space. 
(a)

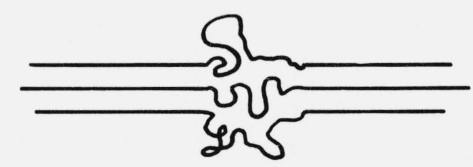

(b)

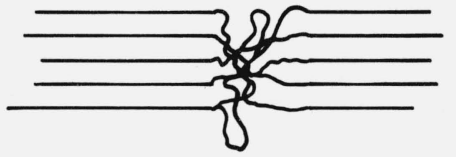

FIGURE 1. Model of Pennings shish proposed by Hoffman (a) after a few chains have been laid down (b) after many chains are laid down. Figure (b) shows the interference possible in the amorphous regime.

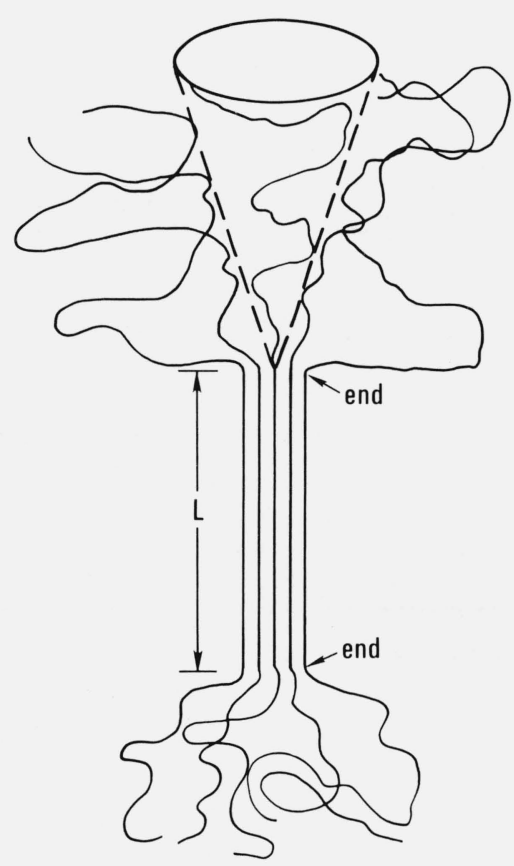

(2a)

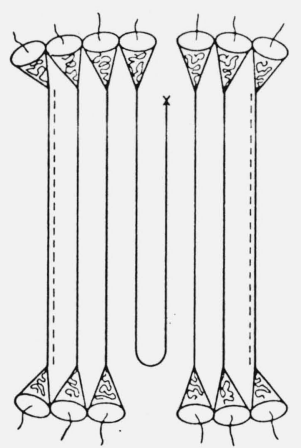

(2b)

FIGURE 2. A polymer crystal of length $L$ and radius $r$. Five cilia emanate from the crystal. (a) A phantom cone, shown by a dotted line, surrounds the central chain to display the effect of interference from the remaining chains on this central chains' configurations. (b) Each cilia in the amorphous regime surrounded by a cone of $Q / n_{c}$.
This competition for space of each chain with the rest causes a reduction in the allowed number of configurations the chain would have if it were free (random coil). We estimate this configurational reduction due to interference by viewing each chain as effectively confined to a smaller cone of angle $\Omega / n_{c}$ by the other chains (see fig. 2a). This assumption is made due to our inability to solve the $n_{c}$ chain problem. A partial justification for such a procedure is given in a subsequent paper [12].

In the next section we shall set up and solve the problem of a chain constrained to remain in a cone. In section 3 we shall use the results of section 2 to estimate the free energy reduction on the surface of the crystal as a function of crystal size.

\section{Solution to the problem of a polymer in a cone}

The problem of polymers constrained to remain in different geometries has been discussed before [1-3]. Recently, Lauritzen and DiMarzio [1] have discussed the problem of a polymer confined to a wedge. In this section we shall generally follow their approach.

Consider a polymer constrained to remain in a cone with absorbing boundary conditions (see fig. 3 ). The axis of the cone is placed along the $z$ axis. In spherical coordinates the cone subtends an angle $\theta_{o}$. A chain is placed in the cone.

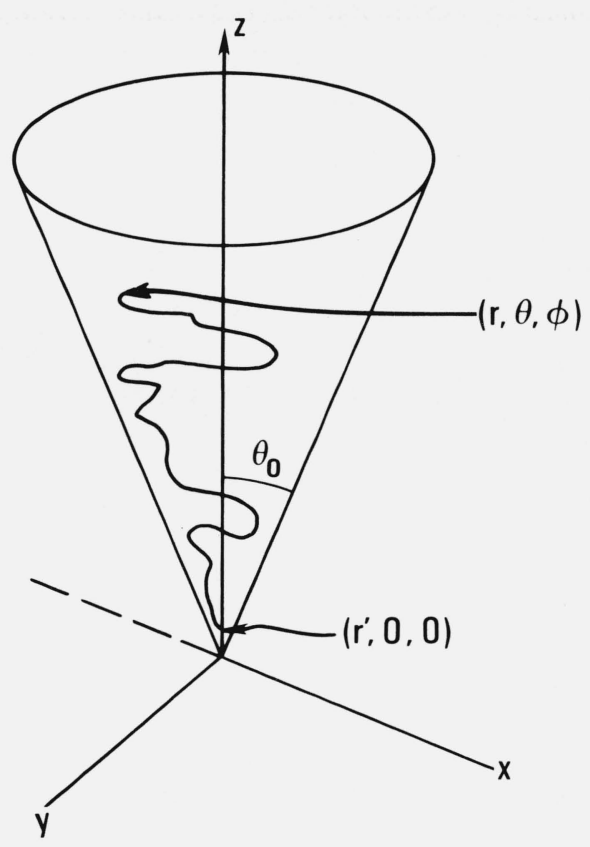

FIGURE 3. Polymer in a cone of angle $\phi_{o}$; cone axis is along the $\mathrm{z}$ axis of the coordinate system. The chain starts at $\left(r^{\prime}, 0,0\right)$ and ends at $(r, \Theta, \phi)$. 
The chain begins at $r=r^{\prime}, \theta=0, \phi=0$. Then following Lauritzen and DiMarzio [1] we can compute $u(r, \theta, \Phi, N)$, the probability density of a chain end being at $(r, \theta, \phi)$ in $N$ steps from the equation

$$
\frac{\partial w}{\partial N}=D \nabla^{2} w
$$

with the boundary conditions $w=0$ on the cone surface $\left(\theta=\theta_{0}\right)$

$$
w(N=0)=\delta\left(r-r^{\prime}, \theta, \phi\right)
$$

where

$$
D=\ell^{2} / 6
$$

$\ell^{2}$ is the expected square of the length of the individual step, $\ell$, and $\delta$ is the three-dimensional Dirac Delta function. The above boundary condition on the cone surface means the cone surface annihilates any polymer which touches it. Note that normalization is not preserved because the annihilated chains are, after the fact of their annihilation, not counted in the integration over space.

In the absence of the cone the partition function for a polymer of $N$ segments is

$$
Q_{o}=\gamma^{N}
$$

where $\gamma$ is the partition function per segment. Then following Lauritzen and DiMarzio [1] the partition function in the presence of the cone is

$$
Q=\gamma^{N} \int_{\text {cone }} w d v
$$

where the integration is over the volume of the cone. Returning to eq (1) and writing it in spherical coordinates we have

$$
\begin{gathered}
\frac{1}{D} \frac{\partial w}{\partial N}=\frac{\partial^{2} w}{\partial r^{2}}+ \\
\frac{2}{r} \frac{\partial w}{\partial r}+\frac{1}{r^{2}} \frac{\partial}{\partial \mu}\left(1-\mu^{2}\right) \frac{\partial w}{\partial \mu}+ \\
\frac{1}{r^{2}}\left(1-\mu^{2}\right) \frac{\partial^{2} w}{\partial \phi^{2}}
\end{gathered}
$$

where $\mu=\cos \theta$

$$
\begin{gathered}
w=\delta\left(r-r^{\prime}, \theta, \phi\right) \text { at } \mathrm{N}=0 \\
w=0 \text { at } \theta=\theta_{0}
\end{gathered}
$$

which is identical to the equation for heat flow in a cone with surface maintained at zero temperature. Such an equation is solved by Carslaw and Jaeger [13]. Their solution is

$$
\begin{gathered}
w=-\frac{1}{4 \pi D N \sqrt{r r}{ }_{v}} \sum_{v}\left(\exp \left(-\left(r^{2}+\left(r^{\prime}\right)^{2}\right) / 4 D N\right)\right) \\
\times(2 v+1) \times I_{v+1 / 2}\left(\frac{r r^{\prime}}{2 D N}\right) P_{v}(\mu) \times \\
{\left[\left(1-\mu_{0}^{2}\right)\left(\frac{d}{d \mu} P_{v}(\mu)\right)_{\mu=\mu_{0}} \cdot\left(\frac{d}{d v} P_{v}\left(\mu_{0}\right)\right)\right]^{-1}}
\end{gathered}
$$

where the sum $\sum_{v}$ is taken over the roots of the equation

$$
P_{v}\left(\mu_{0}\right)=0
$$

and where $I_{v+1 / 2}(\chi)$ is the Modified Bessel function of fractional order.

$P_{v}(\mu)$ is the Legendre function of non-integer order. In the equation in Carslaw and Jaeger [13] (their equation 6 page 384) we have let

$$
\begin{aligned}
& w=v \\
& D=k \\
& N=\tau
\end{aligned}
$$

Notice that we have considered only the solution for a chain starting on the $z$ axis at $\left(r^{\prime}, 0,0\right)$ and where the radial extent of the cone goes to infinity faster then $N$. Other cases have been considered by Carslaw and Jaeger but are not considered here.

Then

$$
\frac{Q}{Q_{0}}=\int_{0}^{\infty} r^{2} d r \int_{\mu_{0}}^{1} d \mu \int_{0}^{2 \pi} d \phi w
$$

The integration on $\phi$ is immediate; for the integration on $\mu$, we have

$$
\begin{gathered}
\frac{Q}{Q_{0}}=+\frac{1}{2 D N} \sum_{v} g(v) \int_{0}^{\infty} \frac{1}{r r^{\prime}} I_{v+1 / 2}\left(\frac{r r^{\prime}}{2 D N}\right) r^{2} d r \\
\times e^{-\left(r^{2}+(r,)^{2}\right) / 4 D N}
\end{gathered}
$$

where

$$
g(v)=\frac{-(2 v+1) \int_{\mu_{0}}^{1} P_{\nu}(\mu) d \mu}{\left(1-\mu_{0}^{2}\right)\left(\frac{d}{d \mu} P_{\nu}(\mu)\right)_{\mu=\mu_{0}} \cdot\left(\frac{d}{d v} P_{\nu}\left(\mu_{0}\right)\right)}
$$

and $v$ runs over the roots of the equation

$$
P_{v}\left(\mu_{0}\right)=0
$$


Two other forms of $g(v)$ shall be useful later. From Carslaw and Jaeger (page 243) [13], we obtain the first form

$$
g(v)=\frac{\int_{\mu_{0}}^{1} P_{v}(\mu) d \mu}{\int_{\mu_{0}}^{1}\left(P_{v}(\mu)\right)^{2} d \mu}
$$

By integration by parts on the differential equation for $P_{v}(\mu)$ (see Carslaw and Jaeger, page 243), we obtain

$$
v(v+1) \int_{\mu_{0}}^{1} P_{\nu}(\mu) d \mu=\left(1-\mu_{0}^{2}\right)\left(\frac{d P_{v}}{d \mu}\right)_{\mu=\mu_{0}}
$$

which when substituted into eq (8) gives the second form for $g(v)$,

$$
g(v)=\frac{-(2 v+1)}{v(v+1) \frac{d}{d v} P_{v}\left(\mu_{0}\right)}
$$

For the integration on $r$ we again follow Lauritzen and DiMarzio[1]. If we let

$$
r^{2}=t
$$

then the integral is a Laplace Transform and we have by [15]

$$
\begin{aligned}
& \frac{Q}{Q_{0}}=+\sum_{v} g(v) \frac{\Gamma\left(\frac{3}{2}+\frac{v}{2}\right)}{\Gamma\left(\frac{3}{2}+v\right)} \\
& \times e^{-z / 2} \frac{1}{z^{3 / 4}} M_{-3 / 4, v / 2+1 / 4}(z)
\end{aligned}
$$

where $M_{k, \mu}(z)$ is the Whittaker function with $z=\left(r^{\prime}\right)^{2} / 4 D N$. By [16]

$$
M_{k, \mu}(z)=z^{1 / 2+\mu} e^{-z / 2} \mid \times M\left(\frac{1}{2}+\mu-k, 1+2 \mu ; z\right)
$$

where $M(a, b ; z)$ is a confluent hypergeometric function.

Thus

$$
\begin{aligned}
\frac{Q}{Q_{0}}=+\sum_{v} g(v) \frac{\Gamma\left(\frac{3}{2}+\frac{v}{2}\right)}{\Gamma\left(\frac{3}{2}+v\right)} e^{-z} \times \\
z^{v / 2} M(3 / 2+v / 2 ; v+3 / 2 ; z)
\end{aligned}
$$

As in [1] we check the normalization on $Q$. That is, we let the chain start a long distance away from the origin of the cone. In this case we should find the chain is unaffected by the presence of the cone. Thus, we should obtain $Q=Q_{0}$. By [16] for

$$
z \rightarrow \infty
$$

we have

$$
\frac{Q}{Q_{0}}=+\sum_{v} g(v)
$$

where the sum is taken over the roots of $P_{v}\left(\mu_{0}\right)=0$. We recall

$$
g(v)=\frac{\int_{\mu_{0}}^{1} P_{v}(\mu) d \mu}{\int_{\mu_{0}}^{1}\left\{\left(P_{v}(\mu)\right)^{2} d \mu\right\}}
$$

Now Carslaw and Jaeger [13] have shown that

$$
\int_{\mu_{0}}^{1} P_{v_{i}}(\mu) P_{v_{j}}(\mu) d \mu=\delta_{v_{i} v_{j}} \int_{0}^{1}\left(P_{\nu}(\mu)\right)^{2} d \mu
$$

if the $v_{K}$ are the roots of $P_{v_{k}}\left(\mu_{0}\right)=0$ and where $\delta_{v v^{\prime}}$ is the Kronecker delta. Thus, summed over $k, P_{v_{K}}$ is an orthogonal polynomial on the space $\mu=1$ to $\mu=\mu_{0}$. Thus, we have for any function of $\mu, h(\mu)$

$$
h(\mu)=\sum_{v_{K}} a_{v_{K}} P_{v_{K}}(\mu)
$$

where

$$
a_{v_{K}}=\frac{\int_{\mu_{0}}^{1} h(\mu) P_{v_{K}}(\mu) d \mu}{N_{K}}
$$

and

$$
N_{K}=\int_{\mu_{0}}^{1}\left(P_{v_{K}}(\mu)\right)^{2} d \mu
$$

Then if $h(\mu)=\delta(1-\mu)$

$$
a_{v_{K}}=\frac{P_{v_{K}}(1)}{N_{K}}=1 / N_{K}
$$

since $P_{v_{K}}(1)=1$.

Thus, we have

$$
\int_{\mu_{0}}^{1} h(\mu) d \mu=\sum_{v_{K}} \frac{\int_{\mu_{0}}^{1} P_{v_{K}}(\mu) d \mu}{\int_{\mu_{0}}^{1}\left(P_{v_{K}}(\mu)\right)^{2} d \mu}=1
$$

By eq (14) we have for $z \rightarrow \infty$ 


$$
\frac{Q}{Q_{0}}=1
$$

which shows $Q$ is correctly normalized.

We now wish to consider the partition function for a long chain attached near the vertex of the cone. Consider

$$
z=\left(r^{\prime}\right)^{2} / 4 D N=\left(r^{\prime}\right)^{2} /\left(2 \ell^{2} N / 3\right)
$$

Following Lauritzen and DiMarzio [1], we assume the polymer is attached at $r^{\prime}=\ell$.

Then,

$$
z=\frac{3}{2 N}
$$

For $N$ large we now calculate $Q / Q_{0}$. For $z$ small and positive $v_{K}$ 's, we have

$$
\begin{aligned}
& M(3 / 2+v / 2 ; \mathrm{v}+3 / 2 ; v)=1+ \\
& \frac{(3+v)}{(2 v+3)} z+\frac{(3+v)(5+v)}{2(2 v+3)(2 v+5)} z^{2}+\ldots
\end{aligned}
$$

By eq (13)

$$
\begin{gathered}
\frac{Q}{Q_{0}}=e^{-z} \sum_{v} g(v) \frac{\Gamma(3 / 2+v / 2)}{\Gamma(3 / 2+v)} \times \\
\left(z^{\prime / 2}+\frac{3+v}{2 v+3} z^{\prime 2+1}+\right. \\
\left.\frac{(3+v)(5+v) z^{\frac{v}{2}+2}}{2(2 v+3)(2 v+5)}+\ldots\right)
\end{gathered}
$$

In the limit of very large $N$ we have

$$
\frac{Q}{Q_{0}}=g\left(v_{0}\right) \frac{\Gamma\left(\frac{3}{2}+\frac{v_{0}}{2}\right)}{\Gamma\left(\frac{3}{2}+v_{0}\right)} z^{\nu^{\prime 2}}
$$

where $v_{0}$ is the lowest root of the equation

$$
P_{v_{K}}\left(\cos \mu_{0}\right)=0
$$

Equations (23a) and (23b) are the key results of this section. They hold as long as $N$ is large and the smallest root of eq (24) is positive. (Notice by Carslaw and Jaeger [13] the roots of eq (24) must be greater than $-1 / 2$.)

Before we can discuss $Q / Q_{0}$ further, we need to evaluate the roots of eq (24) as well as $g(v)$. The $v_{K}$ have been evaluated for two limiting cases; $\theta_{0}$ near $\pi$ [17]. We may also easily evaluate $v_{K}$ at $\theta_{0}=\pi / 2$.

\subsection{Evaluation of $\mathbf{Q} / \mathbf{Q}_{0}$ at $\Theta_{0}=\pi / 2$}

By [16] eq 8.6.1

$$
\begin{gathered}
P_{\nu}(\cos \pi / 2)=P_{\nu}(0)=\pi^{-1 / 2} \cos \left(\frac{1}{2} \pi v\right) \\
\times \Gamma\left(\frac{v}{2}+1 / 2\right) / \Gamma\left(\frac{v}{2}+1\right)
\end{gathered}
$$

Therefore, the roots of $P_{v}(\cos \pi / 2)$ for positive $v$ are

$$
v=(2 i+1), i=0,1 \ldots
$$

For $g(v)$ we then have by reference [16] eq 8.14 .13 and 8.14.15

$$
\begin{gathered}
g(1)=3 / 2 \\
g(3)=-7 / 8 \\
g(5)=11 / 16
\end{gathered}
$$

Then, by eq (23a) we have

$$
\begin{aligned}
\frac{Q}{Q_{0}} & =e^{-z} \cdot \frac{\Gamma(2)}{\Gamma(5 / 2)}\left(\frac{3}{2}\right) z^{1 / 2} \\
+ & e^{-z} \cdot \frac{\Gamma(2)}{\Gamma(5 / 2)}\left(\frac{3}{2} \cdot \frac{4}{5}\right) z^{3 / 2} \\
& +e^{-z}(-7 / 8) \frac{\Gamma(3)}{\Gamma(9 / 2)} z^{3 / 2}
\end{aligned}
$$

to powers of $z^{3 / 2}$. Expanding $e^{-z}$ we have

$$
\frac{Q}{Q_{0}}=\frac{2}{\pi^{1 / 2}} z^{1 / 2}-2 / 3 \frac{z^{2 / 3}}{\pi^{1 / 2}}
$$

which is identical to the Lauritzen and DiMarzio [1] result for a wedge with $\alpha=\pi$ as one would expect.

\subsection{Evaluation of $Q / Q_{0}$ for $\Theta_{0}$ near $\pi / 2$}

We now shall look at the region around $\pi / 2$ to see if we still obtain the Lauritzen and DiMarzio result. Let $\Theta_{0}=$ $\pi / 2-\varepsilon$ where $\varepsilon$ is small. Then

$$
\begin{aligned}
& P_{v}\left(\cos \Theta_{0}\right)=P_{v}(\cos \pi / 2)- \\
& \varepsilon \sin (\pi / 2)\left(\frac{d P_{v}}{d \cos \Theta_{0}}\right)_{\Theta_{0}=\pi / 2}
\end{aligned}
$$

By [16] (8.6.1) and (8.6.3) we have

$$
\begin{gathered}
P_{v}\left(\cos \Theta_{0}\right)=\pi^{-1 / 2} \cos \left(\frac{\pi}{2} v\right) \Gamma\left(\frac{v}{2}+1 / 2\right) / \Gamma\left(\frac{v}{2}+1\right) \\
-2 \varepsilon \pi^{-1 / 2} \sin \left(\frac{\pi}{2} v\right) \Gamma\left(\frac{v}{2}+1\right) / \Gamma\left(\frac{v}{2}+1 / 2\right)
\end{gathered}
$$


For the root we have $P_{v}\left(\cos \Theta_{0}\right)=0$ and thus the roots $v_{i}$ are determined by the implicit equation

$$
\varepsilon=\frac{\cos \left(\frac{\pi}{2} v_{i}\right)}{2 \sin \left(\frac{\pi}{2} v_{i}\right)}\left(\frac{\Gamma\left(\frac{v_{i}}{2}+1 / 2\right)}{\Gamma\left(\frac{v_{i}}{2}+1\right)}\right)^{2}
$$

Now the roots for $\varepsilon=0$ are given by

$$
v_{i}^{0}=(2 i+1) i=0,1,2, \ldots
$$

If we take each

$$
v_{i}=v_{i}^{0}+\tau_{i}
$$

we may expand the right hand side of eq (31) and obtain to first order

$$
\tau_{i}=\varepsilon\left(\frac{\Gamma\left(\mathrm{i}+\frac{3}{2}\right)}{\Gamma(\mathrm{i}+1)}\right)^{2} 4 / \pi
$$

and we obtain

$$
\begin{gathered}
\tau_{0}=\varepsilon \\
\tau_{1}=\frac{9}{4} \varepsilon
\end{gathered}
$$

or

$$
v_{0}=1+\varepsilon ; v_{1}=3+(9 / 4) \varepsilon
$$

Then by eq(23) we have

$$
\frac{Q}{Q_{0}} \propto z^{(1+\varepsilon) / 2}
$$

To compare this result to the formula of Lauritzen and DiMarzio for a wedge we replace $\alpha=\pi-2 \varepsilon$ in the formula for $Q / Q_{0}$ to obtain

$$
\frac{Q}{Q_{0}} \propto z^{n / 2 \alpha} \propto z^{(1+2 \varepsilon / \pi) / 2}
$$

Thus, for just a small deviation from $\pi / 2$ the cone and the wedge differ to the first order.

\subsection{Evaluation of $Q / Q_{0}$ near $\Theta_{0}=0$}

MacDonald [17] has shown for $\Theta$ small the roots of $P_{v_{i}}(\cos$ $\Theta)$ are

$$
v_{i}=\chi_{i} / \Theta
$$

$\chi_{i}$ are the roots of $J_{0}(x)$, the Bessel function of zeroth order. These roots are by [16] page 409

$$
\begin{aligned}
& \chi_{0}=2.4048 \\
& \chi_{1}=5.2007 \\
& \chi_{2}=8.637
\end{aligned}
$$

Thus, by eq (23) for small $\Theta_{0}$

$$
\frac{Q}{Q_{0}}=g\left(\frac{\chi_{0}}{\Theta_{0}}\right) \frac{\Gamma\left(\frac{3}{2}+\frac{\chi_{0}}{2 \Theta_{0}}\right)}{\Gamma\left(\frac{3}{2}+\frac{\chi_{0}}{\Theta_{0}}\right)} \times z^{\chi_{0} / 2 \theta_{0}}
$$

This is to be compared to Lauritzen and DiMarzio [1] eq (28) with $\alpha=2 \Theta_{0}$. Their result is

$$
\frac{Q}{Q_{0}} \propto z^{n / 4 \theta_{0}}
$$

Here we see that there are differences between the wedge and the cone at small angles. However, as before, the general character of the partition function is similar.

\subsection{Evaluation for $\Theta$ near $\pi$}

MacDonald [17] has obtained the roots of $P_{v}\left(\cos \Theta_{0}\right)$ near $\Theta_{0}=\pi$. He obtains

$$
v_{K}=k+\frac{1}{2 \log \left(2 /\left(\pi-\Theta_{0}\right)\right)}
$$

where

$$
k=0,1,2, \ldots
$$

Thus, by eq (23) we obtain for first order in $z$

$$
\frac{Q}{Q_{0}}=g\left(v_{0}\right) \cdot \frac{\Gamma\left(\frac{3}{2}+\frac{v_{0}}{2}\right)}{\Gamma\left(\frac{3}{2}+v_{0}\right)} z^{\nu_{0}}
$$

where

$$
v_{0}=\frac{1}{2 \log \left(2 /\left(\pi-\Theta_{0}\right)\right.}
$$

As $\Theta_{0}$ approaches $\pi, v_{0}$ gets small but remains positive and $\mu_{0}$ approaches -1 . Thus

$$
g\left(v_{0}\right)=\frac{\int_{-1}^{+1} P_{0}(\mu) d \mu}{\int_{-1}^{+1} P_{0}(\mu)^{2} d \mu}=1
$$

and 


$$
\frac{Q}{Q_{0}}=1
$$

as one would expect for a infinite sphere. This result differs from the Lauritzen and DiMarzio [1] result at $\alpha=2 \pi$. This too is expected since in the limit of $\alpha$ going to $2 \pi$ a wedge is a half plane while an infinite sphere (an infinite cone at $\pi$ ) is equivalent to free space.

\subsection{Evaluation of $Q / Q_{0}$ roots of $\mathbf{P}_{v}(\cos \Theta)$ for any general angle between 0 and $\Pi$}

The two lowest roots of the Legendre function were obtained by determining the roots of the polynomial

$$
0=P_{v}\left(\cos \Theta_{0}\right)=\sum_{n=0}^{\infty} \frac{(a)_{n}(b)_{n} t^{n}}{(n !)^{2}}
$$

where

$$
\begin{gathered}
(d)_{n}=d(d+1) \ldots(d+n-1) \\
(d)_{0}=1 \\
t=\left(1-\cos \Theta_{0}\right) / 2 \\
a=-v \\
b=v+1 \\
c=1
\end{gathered}
$$

(see [16] eq 8.1.2 and 15.1.1). These roots are given in intervals of $\pi / 24$ in table I. Also given are values of $g(v)$ for the lowest root evaluated using eq $(10 \mathrm{~b})$ with a numerical differentiation for

$$
\frac{d}{d v} \quad P_{v}\left(\mu_{0}\right)
$$

TABLE 1. For $\theta_{0}=J \pi / 24$

\begin{tabular}{rrrr}
\hline \hline $\mathrm{J}$ & \multicolumn{1}{c}{$v_{0}$} & $v_{1}$ & $g\left(v_{0}\right)$ \\
\hline 1 & 17.869 & 41.669 & 1.60 \\
2 & 8.681 & 20.583 & 1.60 \\
3 & 5.617 & 13.554 & 1.60 \\
4 & 4.084 & 10.039 & 1.59 \\
5 & 3.163 & 7.929 & 1.58 \\
6 & 2.548 & 6.522 & 1.58 \\
7 & 2.108 & 5.517 & 1.57 \\
8 & 1.777 & 4.763 & 1.56 \\
9 & 1.519 & 4.176 & 1.54 \\
10 & 1.312 & 3.706 & 1.53 \\
11 & 1.142 & 3.321 & 1.52 \\
12 & 1.000 & 3.000 & 1.50 \\
13 & 0.879 & 2.728 & 1.48 \\
14 & .774 & 2.494 & 1.46 \\
15 & .683 & 2.291 & 1.44 \\
16 & .602 & 2.113 & 1.42 \\
17 & .529 & 1.955 & 1.39 \\
18 & .463 & 1.813 & 1.37 \\
\hline
\end{tabular}

In figure 4 we plot $v_{0} \Theta_{0}$ versus $\Theta_{0}$. It is seen from this that we may approximate $v_{0}$ as

$$
\begin{gathered}
v_{0}=\frac{A\left(\Theta_{0}\right)}{\Theta_{0}} \\
A\left(\Theta_{0}\right)=2.4048-0.5000\left(\Theta_{0}\right)-0.025\left(\Theta_{0}\right)^{2} \\
\text { for } 0<\Theta_{0}<2 \pi / 3
\end{gathered}
$$

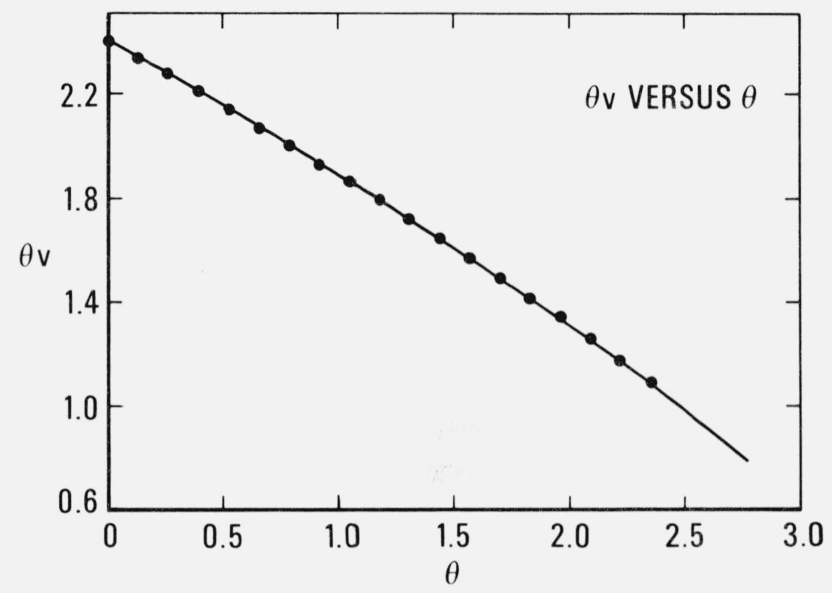

FIGURE 4. Plot of $v_{0} \Theta_{0}$ versus $\Theta_{0}$. Points are calculated from eq (46). Curve is eq (47b) line.

Similarly in figure $5 g\left(v_{0}\right)$ is plotted versus $\Theta_{0}$ and we have

$$
\begin{gathered}
g\left(v_{0}\right)=\mathrm{B}\left(\Theta_{0}\right)=1.603-0.0425\left(\Theta_{0}\right)^{2} \\
0 \leqslant \Theta_{0} \leqslant 2 \pi / 3
\end{gathered}
$$

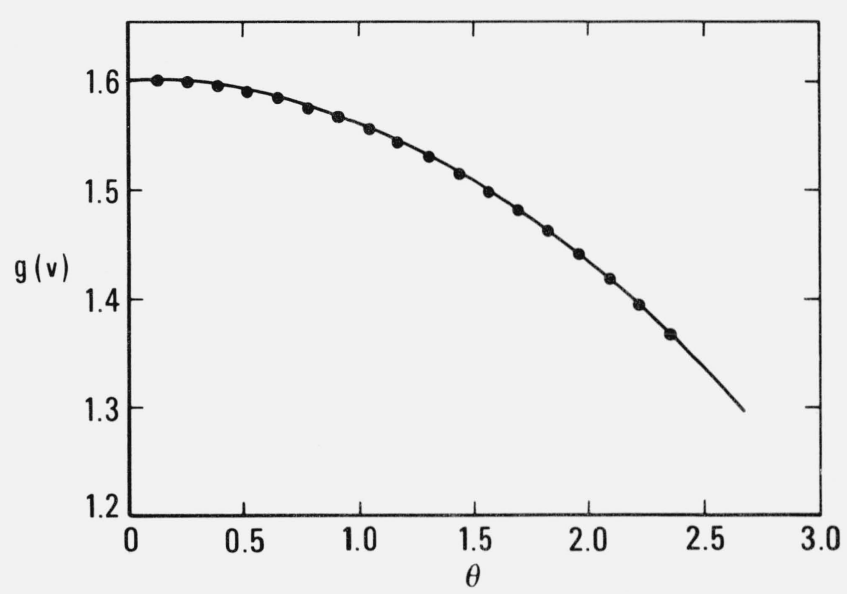

FIGURE 5. Plot of $g\left(v_{\alpha}\right)$ versus $\Theta_{0}$ from eq (48). 
Thus, by eq (23b) we have

$$
\begin{gathered}
\ln Q / Q_{0}=\ln B\left(\Theta_{0}\right)+\ln \left\{\frac{\Gamma\left(\frac{3}{2}+\frac{A\left(\Theta_{0}\right)}{2 \Theta_{0}}\right)}{\Gamma\left(\frac{3}{2}+\frac{A\left(\Theta_{0}\right)}{\Theta_{0}}\right)}\right\} \\
+\frac{A\left(\Theta_{0}\right)}{2 \Theta_{0}} \ln (3 / 2 N)
\end{gathered}
$$

For $\theta_{0}$ small, we have

$$
\ln Q / Q_{0}=\ln (.8)+\frac{v_{0}}{2}-\frac{v_{0}}{2} \ln \left(\frac{v_{0} N}{3}\right)
$$

or

$$
\ln Q / Q_{0} \cong-.22-\frac{1.202}{\Theta} \ln \left(.295 \frac{N}{\Theta}\right)
$$

\section{A Model for the Calculation of Surface Free Energy}

We now wish to use the results of the previous section to estimate the surface free energy (or phase boundary free energy) when many cilia emerge from a surface of a polymer crystal. In figures $2 \mathrm{a}$ and $2 \mathrm{~b}$ we show a model of the crystallite of the form we are considering. The chains of the crystal are extended along the $\mathrm{L}$ direction. The chains emanate from the end surface of the crystal and become random coils. However, one would expect interference in such a system. In order to estimate the free energy due to the interference, we model space as in figure $2 b$ where the space around the crystal is divided into equal cones, the number of cones being equal to the number of emanating chains. Thus, the interference is taken into account by requiring each chain to remain in its own cone. Models such as these would be expected to be useful for calculations of the surface free energy for a fringed micelle model or on a model of the shish-kebob crystal as proposed by Hoffman [9] and by Keller [10].

The estimation of the end surface free energy follows. Let us say $\eta$ cilia per unit area emanate from the crystal on the end surface. Then for a crystal of radius $r, 2 \pi r^{2} \eta$ cilia come from total crystal (two surfaces). The size of the crystal is small compared to the volume occupied by the cilia. Thus each cilia cuts a cone of angle, $\theta$, where we assume a sphere surrounding the crystallite may be divided into $2 \pi r^{2} \eta$ cones. We assume each cilia is constrained to remain in its own cone. Thus we have for $\theta$

$$
\theta=4 \pi / 2 \pi r^{2} \eta=\frac{2}{r^{2} \eta}
$$

The above formula disregards the interference from the crystal itself. But for the cilia length large and $L$ small we may expect this assumption to be valid. The major approximation in the above is the assumption that the interference of one cilia with the rest of its neighbors may be estimated by containing it in the fraction of volume available to it in the sphere (a cone of angle $\theta$ ). The justification for this assumption will be amplified in a later paper [12]. For now let us say this approach is similar to van der Waals theory of the liquid state where the reduction in number of configurations available to a molecule is accounted for by the condition that the volume available to that molecule of the liquid is very closely its molecular specific volume.

By the results of the previous section the free energy difference between one polymer of length $N$ in a cone and a random coil chain unconstrained of length $N$ is

$$
\Delta f=-k_{B} T \ln Q / Q_{0}
$$

Thus, from eqs (49a), (50) and (51) we obtain the free energy reduction of a cilia in the above model.

The free energy of the total interface is simply the sum of the contributions from each cilia

$$
\Delta F=2 \pi r^{2} \eta \Delta f
$$

It is interesting to look at the free energy when many chains emanate from the end surface, that is, when $\pi r^{2} \eta$ is large. In this case $\Theta$ is small and the equations for the free energy become particularly simple. Thus, we may use eq (49b) and obtain for $\Delta F$

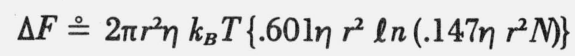

Taking the total interfacial free energy as the surface free energy, we have, for the end surface energy per unit area, $\sigma_{e}$

$$
\sigma_{e} \stackrel{=}{=} k_{B} T\left(.601 \eta r^{2} \ln \left(.147 \eta r^{2} N\right)\right)
$$

Thus the surface free energy, $\sigma_{e}$, increases faster than the surface area. In fact for fixed $L$, the surface free energy per unit area grows faster than the volume of the crystal, and the surface free energy will quickly exceed the bulk free energy.

Some examples of this previous point would be instructive. We start by computing $\sigma_{e}$. Consider a crystallite of polyethylene (PE) of $100 \AA$ radius at $300 \mathrm{~K}$. Thus with

$$
\eta=\frac{f}{18.9} \times 10^{+10} \text { for } \mathrm{PE}[18]
$$


and

$$
\begin{gathered}
k_{B} T=414 \times 10^{-16} \mathrm{ergs} \\
\eta k_{B} T=21.9 f \\
\eta r^{2}=529 f
\end{gathered}
$$

where $f$ is the fraction of crystal stems which have emergent cilia attached ( $1-f$ is the fraction of crystal stems ending or having folds). We may now compute $\sigma_{e}$ for these parameters and a cilia length of 5 statistical units and 50 statistical units (for PE 5 statistical units is about 35 segments). We allow $f$ to vary from 1 (case of no folds) to 0.02 (case of $98 \%$ folds).

Values of $\sigma_{e}$ are given in table 2. For comparison the fold surface free energy in polyethylene is $90 \mathrm{ergs} / \mathrm{cm}^{2}$ [18] while the surface free energy of an alkane crystal is about $12 \mathrm{ergs} / \mathrm{cms}^{2}[18,19]$. We see the surface free energy varies as a function of both $f$ and $N$ and it can

\begin{tabular}{|c|c|}
\hline \multicolumn{2}{|c|}{$\mathrm{N}=5$} \\
\hline$f=$ & $\sigma_{e}$ calculated ergs $/ \mathrm{cm}^{2}$ \\
\hline 1 & 41,374 \\
\hline 0.1 & 254 \\
\hline .067 & 101 \\
\hline .05 & 51 \\
\hline .04 & 30 \\
\hline \multicolumn{2}{|c|}{$\mathrm{N}=50$} \\
\hline$f=$ & $\sigma_{e}$ calculated $\mathrm{ergs} / \mathrm{cm}^{2}$ \\
\hline 0.1 & 4.14 \\
\hline .067 & 173 \\
\hline .05 & 91 \\
\hline .04 & 56 \\
\hline .021 & 13.5 \\
\hline
\end{tabular}
become very large.

TABLE 2.

Now let us show that the surface free energy may become so large that it may well overwhelm the bulk free energy. To see this, we consider a crystallite of $\mathrm{PE}$ of length $200 \AA(L=200 \AA)$ at 40 degrees below its melting point of $420 \mathrm{~K}$. We then ask, for this case, at what $r$ will the surface free energy overwhelm the bulk free energy of crystallization. The bulk free energy of crystallization is given by [18]

and

$$
\Delta f_{c}=\Delta h \frac{\Delta T}{T_{m}} 4 \pi r^{2} L
$$

$$
\frac{\Delta f_{c}}{r^{2}}=7 \times 10^{3}
$$

where we have assumed

$L=200 \AA$

$\Delta T=40$

$T_{m}=420 \mathrm{~K}$

$\Delta h=2.8 \times 10^{9} \mathrm{ergs} / \mathrm{cm}^{3}(\mathrm{see}[18])$

Equation 53 gives the surface free energy, $\Delta F$. Consider the case of a chain of 50 statistical units $(N=50)$ and $T=380$ $\mathrm{K}\left(T=T_{m}-\Delta T\right)$. Then

$$
\frac{\Delta F}{r^{2}}=5.54 r^{2} f^{2} \cdot 10^{16} \ln \left(.39 f r^{2} 10^{16}\right)
$$

Tables 3 gives $\Delta F$ as a function of $r$ for two values of $f ; f=1$ (no folding) and $f=.33$ ( $2 / 3$ folding).

For $\mathrm{f}=1$ at a radius of $15 \AA$ for the crystal the free energy of the surface becomes larger than that of the bulk and we would expect the crystal to change at this radius. For $f=.33$ the bulk free energy is overwhelmed by the surface free energy at about a radius of $50 \AA$.

TABLE 3 .

\begin{tabular}{rcc}
\hline & & $\Delta \mathrm{F}$ in ergs/cm \\
$r$ in $\AA$ & $\mathrm{f}=1$ & $\mathrm{f}=.33$ \\
\hline 10 & $2 \times 10^{3}$ & $1.5 \times 10^{2}$ \\
20 & $11 \times 10^{3}$ & $1 \times 10^{3}$ \\
30 & $\sim$ & $2.6 \times 10^{3}$ \\
40 & $\sim$ & $5.1 \times 10^{3}$ \\
50 & $\sim$ & $8.7 \times 10^{3}$ \\
100 & $\sim$ & $43 \times 10^{3}$ \\
\hline
\end{tabular}

Thus, we see that for highly ciliated surfaces the contribution to the free energy due to cilia interference, on the surface, as estimated from this model, can easily exceed the bulk free energy at crystallization and would thus be expected to play a dominate role in the crystal thermodynamics as well as the crystal kinetics $[9,18]$.

The observation that total surface free energy can overwhelm the free energy of crystallization allows one to construct a theory of crystal growth in which there is an optimum crystal size due to the accumulation of surface strains (strain limited growth). This has been used by one of us [9] to explain the shish-kebob morphology of polymers crystallized by Pennings [11].

However, the existence of lamellar polymer crystals means that eq (54) must finally fail for large $r$. There are two ways this can happen. (1) Chain folding can occur while the crystal is small. This relieves the strains along the surface because now fewer amorphous chains per unit area compete for the space above the surface [7]. Then the crystal grows beyond a size where this model and eq $(54)$ is appropriate to 
calculate the interference effects. (2) From another point of view the amorphous chains may orient as the crystal grows. This would allow the crystal to "eat" into the amorphous regime until the amorphous region disappears.

\section{References}

[1] Lauritzen, J. I., and DiMarzio, E. A., The Configurational Statistics of a Polymer Confined to a Wedge of Interior Angle $\alpha$, J. Res. Nat. Bur. Stand. (U.S.), Vol. 83, No. 4, 381-385 (July-Aug. 1978).

[2] DiMarzio, E. A. and Rubin, R. J., J. Chem. Phys. 55, 4318 (1971).

[3] Gaylord, R. J. and Lohse, D. J., Bull. Am. Phy. Soc., 23372 (March 1978).

[4] Helfand, E. and Tagami Y., J. Chem. Phys. 56, 3592 (1972).

[5] Dolan, A. K. and Edwards, S. F., Proc. Roy. Soc. London A 337, 509 (1974).

[6] Flory, P. J., J. Am Chem. Soc., 842857 (1962).

[7] DiMarzio, E. A., Bull, Am. Phys. Soc., 23420 (March 1978).
[8] Guttman, C. M. and DiMarzio, E. A. Bull. Am. Phys. Soc., 25, 318 (1980).

[9] Hoffman, J. D., Polymer, 201071 (1979).

[10] Grubb, D. T. and Keller, A., Colloid Polym. Sci., 256, 218 (1978).

[11] Pennings, A. J., J. Poly. Sci., Poly. Sym. 59, 55 (1977).

[12] DiMarzio, E. A., Guttman, C. M., and Hoffman, J. D. (in preparation).

[13] Carslaw, H. S. and Jaeger, J. C., Conduction of Heat in Solids, Chapter IX and XIV, (Oxford Univ. Press, 1959).

[14] Hobson, E. W., Theory of Spherical and Ellipsodal Harmonics, (Chelsea Publ. Co., 1955).

[15] Bateman, H., Bateman Manuscript Project, Table of Integral Transforms, Vol. 1, (McGraw Hill, 1954) p. 197.

[16] Abramowitz, M. and Stegun, I. A., Handbook of Mathematical Functions, NBS Applied Math Series \#55 (1964).

[17] MacDonald, H. M., Proc. London Math. Soc., 31, 264 (1899).

[18] Hoffman, J. D., Davis, G. T., and Lauritzen Jr., J. I., Treatise on Solid State Chemistry, Bruce Hannay ed., (Plenum Press, N.Y., 1976), vol. 3, chap. 7 .

[19] Turnbull, D and Spaepen, F., J. Poly. Sci., Polymer Sym. 63, 237 (1978). 